\title{
Maternal separation can affect the reproductive system by inflammasome activation in female mice
}

\author{
Kajal Khodamoradi, a, bossein Amini-Khoei, ${ }^{,}$Zahra Khosravizadeh, ${ }^{a}$ Seyed Reza Hosseini, ${ }^{d}$ \\ Ahmad Reza Dehpour, and Gholamreza Hassanzadeha*
}

\author{
aDepartment of Anatomy, School of Medicine, Tehran University of Medical Sciences, Tehran, Iran. \\ bMiller School of Medicine, University of Miami, Miami, FL, USA. \\ 'Medical Plants Research Center, Basic Health Sciences Institute, Shahrekord University of Medical Sciences, Shahrekord, Iran. \\ dDepartent of Urology, School of Medicine, Tehran University of Medical Sciences, Tehran, Iran. \\ experimental Medicine Research Center, Tehran University of Medical Sciences, Tehran, Iran. \\ ${ }^{*}$ Correspondence to Gholamreza Hassanzadeh (email: hassanzadeh@tums.ac.ir). \\ (Submitted: 04 December 2018-Revised version received: 16 January 2019 - Accepted: 23 April 2019 - Published online: 26 June 2019)
}

\begin{abstract}
Objective The aim of this study is to investigate effect of maternal separation stress on the ovarian function in adult female mice.
Methods In this study, maternal separation in pups was performed during post-natal days 2-14. The histological alterations in ovarian tissue, reactive oxygen species (ROS) production (using 2',7'-dichlorofluorescin diacetate assay), gene expression of NLRP3, apoptosisassociated speck-like protein containing a CARD (ASC), caspase-1, TLR4, BAX, BCL-2 and TNF-a (using RT-PCR), protein levels of ATP, GPx, interleukin (IL)-1 $\beta$ and IL-18 (using enzyme-linked immunosorbent assay). Also, protein expression of caspase-3 and NLRP3 (using immunocytochemistry) were evaluated.

Results This showed that maternal separation decreased percentage of primordial follicles while increased percentage of secondary and Graafian follicles. In addition, maternal separation increased ROS production and decreased ATP and GPx concentrations. Furthermore, maternal separation significantly affected expression of cytokines and genes involved in inflammation and apoptosis including NLRP3, ASC, caspase-1, TLR4, TNF-a, IL-1 $\beta$, IL-18, BAX and BCL2. Findings also showed that stress-induced maternal separation significantly increased percentage of caspase-3 and NLRP3 positive cells. We concluded that maternal separation stress has harmful effects on ovarian tissue.

Conclusion It seems that these harmful effects probably occur through increase of ROS production and impact on mitochondrial function, inflammatory process and apoptosis pathways.

Keywords maternal separation stress, inflammasome, inflammation, oxidative stress, apoptosis, reproductive system
\end{abstract}

\section{Introduction}

The early childhood is a sensitive age period in which development of brain can be affected harmfully by stressful events. The maternal separation model, as a model mimicking early-life stress, has been broadly studied to evaluate the effect of early-life stress on brain development and associated biological networks. Exposure to this stressful event can affect children not only in their early years of life but also in their adult life. It seems that the repeated activation of the hypothalamicpituitary adrenal (HPA) axis and disruptions in pathways of the immune response and the neurotrophin production could play a critical role in the long-lasting effects of early-life stress. ${ }^{1,2}$

Stress can trigger the activity of the adrenergic system that change the function of the endocrine and immune systems. ${ }^{3}$ Cooperation between the immune and endocrine systems is necessary for normal development and function of the female reproductive system. ${ }^{4}$

According to psychoneuroimmunology studies, the physiological response to stressors affects function of the innate immune system even in the absence of pathogens that is known as sterile inflammatory response. ${ }^{5,6}$ Although it remains unknown how innate immune function is stimulated by stress, the role of different factors including glucocorticoids, danger signals and catecholamines are evidenced by several studies. ${ }^{7-10}$ The transcription of inflammatory mediators such as cytokines and chemokines are activated during the inflammatory process. ${ }^{11}$ Inflammasomes are intracellular multi-protein complexes which include a NOD-like receptor/an AIM-like receptor, the adapter molecule apoptosis-associated speck-like protein containing a CARD (ASC), and caspase- $1 .{ }^{12-14}$ NLRP3 along with the adaptor protein ASC, active caspase-1 through assembly of the inflammasome, although the exact mechanism for this process is unclear. The NLRP3, the most described inflammasome, can be triggered by a wide range of exogenous and endogenous stress signals. Caspase- 1 activation leads to the cleavage of pro-interleukin (IL)-1beta, pro-IL-18 into active forms of IL-1 $\beta$, IL-18, which play potential role in modulating innate immune function. ${ }^{15-17}$ Moreover, caspase- 1 plays a role in cell apoptosis via stimulation of caspase- 3 and $-7 .{ }^{18}$

On the other hand, psychosocial stressors via stimulation of inflammatory cytokines such as TNF- $\alpha$, IL1- $\beta$ and IL- 6 can lead to the suppression of gonadotropin-releasing hormone (GnRH) secretion. ${ }^{19}$ Since the main target of $\mathrm{GnRH}$ is the gonadotropes of anterior pituitary gland, stress-induced GnRH suppression can affect the secretion of gonadotropic hormones and consequently reproductive functions.

Mitochondria are responsible for the reactive oxygen species (ROS) production, ATP generation and apoptotic control. ${ }^{20}$ Owing to the high levels of antioxidant enzymes, the mitochondria can regulate the balance between ROS production and degradation. ${ }^{21}$ The findings of studies have confirmed that chronic stress can lead to disruption of mitochondrial function and free radicals production..$^{22}$ It is apparent that mitochondria play a significant role in female reproductive processes including oocyte maturation, fertilization and early embryogenesis. ${ }^{23}$ The evidence for the involvement of oxidative injury in the 
pathogenesis of infertility in females has been accumulating since long. ${ }^{24}$

Since the adverse effects of early-life stress on the neuroendocrine and innate immune system, also adverse effects of oxidative stress on fertility status have been well described in previous studies, stress-induced maternal separation during early life may have an adverse effect on the female reproductive system. Therefore, this study was designed to investigate the effect of maternal separation stress on ovarian function in adult female mice.

\section{Materials and Methods}

\section{Experimental Animals}

Pregnant Naval Medical Research Institute mice were obtained from the Pasteur Institute of Iran. The animals were housed under a cycle of $12 \mathrm{~h}: 12 \mathrm{~h}$ light/dark at controlled temperature $\left(22-25^{\circ} \mathrm{C}\right)$ and humidity (55-65\%). All animal procedures were carried out according to guidelines approved by the Ethics Committee of Tehran University of Medical Sciences (IR.TUMS.MEDICINE.REC.1395.2507). To produce maternal separation model, pups were separated from their mothers and placed in a clean cage for $3 \mathrm{~h}$ every day ( 9 am to $12 \mathrm{pm}$ each day) from post-natal days (PND) 2 to 14. The birthday of pups was considered as PND $0 .{ }^{25-27}$ In this study, female pups were randomly divided into two groups: control and maternal separation group. The pups of the control group were left untouched. Mice at PND 70 were sacrificed by deep anesthesia and the ovaries were removed. One ovary from each mouse was used for histological assessment and the second ovary was used for molecular assessments.

\section{Histological Assessment}

The ovaries for histological assessment were immediately transferred to Bouin's fixative solution. The fixed ovaries were dehydrated in ascending graded series of ethanol (Merck, Darmstadt, Germany) and then emerged in paraffin wax. The serial sections $(5 \mu \mathrm{m}$ thick) were prepared with a rotary microtome (Microm, Walldorf, Germany) and rehydrated in descending graded series of ethanol. The sections were cleared in xylene, stained with hematoxylin and eosin (H\&E) and mounted with DPX. For histological assessment, transverse sections from nine different regions of the ovaries were examined. The number of primordial, primary, secondary and Graafian follicles were counted by light microscopy and ImageJ software (ImageJ U. S. National Institutes of Health, Bethesda, MD, USA).

\section{ROS Assay}

The level concentration of ROS production in ovarian tissues was detected with flow cytometry using $2^{\prime}, 7^{\prime}$-dichlorofluorescin diacetate (DCFH-DA; Sigma-Aldrich, St. Louis, MO, USA) after enzymatic digestion of minced tissue. ${ }^{28}$ The ovarian tissues were mechanically homogenized in Ham's F-10 medium (Life Technology, Carlsbad, CA, USA). The homogenates of ovarian tissue were centrifuged at $10,000 \mathrm{~g}$ for $5 \mathrm{~min}$ and washed with PBS. Then, the homogenates were incubated with $20 \mu \mathrm{M}$ DCFH-DA in dark at $37^{\circ} \mathrm{C}$ for $45 \mathrm{~min}$. The homogenates of ovarian tissue were washed with PBS, and DCF fluorescence (green) was measured in the FL-1 channel using a BD FACScan flow cytometer (Becton Dickinson, San Jose, CA, USA). ${ }^{29}$

\section{Real-time Reverse Transcription Polymerase Chain Reaction Analysis}

The expression level of NLRP3, ASC, caspase-1, TLR4, BAX, BCL2 and TNFa genes was analyzed by real-time reverse transcription polymerase chain reaction (RT-qPCR). The total RNA extraction from ovarian tissues was performed with TRIzol reagent according to the manufacturer's instructions (Invitrogen, Carlsbad, CA, USA). The complementary DNA was produced via reverse transcription reaction using a PrimeScript RT reagent kit (Takara, South Korea) according to the manufacturer's protocol. The RT-qPCR was carried out with gene specific primers and the HOT FIREPol EvaGreen qPCR Mix Plus (Solis BioDyne, Tartu, Estonia) by an ABI7500 (Applied Biosystems, Foster City, CA, USA). The reference gene glyceraldehydes-3-phosphate dehydrogenase messenger RNA (mRNA) expression are used as the internal control to normalize mRNA expression levels. The level of target genes expression was calculated using $2^{-\Delta \mathrm{CT}}$. List of primer sequences used for RT-qPCR analysis are presented in Table 1.

\section{Enzyme-linked Immunosorbent Assay}

The ovarian tissues were homogenized in PBS on ice, then centrifuged at $800 \times \mathrm{g}$ for $5 \mathrm{~min}$. The collected supernatants were used in the enzyme-linked immunosorbent (ELISA) assay. The level concentrations of ATP and GPx were measured with specific ELISA kits (R\&D Systems, Minneapolis, MN, USA) and the level concentrations of IL- $1 \beta$ and IL-18 in ovarian tissue were measured using ELISA kits (Koma Biotech, Seoul, Korea) according to the manufacturer's protocols.

\section{Immunocytochemical Analysis}

To determine caspase- 3 and NLRP3 immunoreactivity, the fixed ovarian samples were dehydrated in graded ethanol and embedded in paraffin. After removing paraffin, samples were rehydrated through a graded series of ethanol and permeabilized with $10 \mathrm{mM}$ sodium citrate and $0.05 \%$ Tween 20. The samples were blocked in a blocking solution including $1 \%(w / v)$ bovine serum albumin (Sigma-Aldrich, St. Louis, MO, USA) in PBS. Then, the ovarian samples were incubated overnight at $4^{\circ} \mathrm{C}$ with primary antibodies against caspase- 3 (1:1000 dilution, Abcam, Cambridge, MA, USA) and NLRP3

\begin{tabular}{|c|c|c|}
\hline Genes & Forward primers & Reverse primers \\
\hline NLRP3 & $\begin{array}{l}\text { 5'-GGACCCACAGTG- } \\
\text { TAACTTGCAGA-3' }\end{array}$ & $\begin{array}{l}\text { 5'-AGGCTG- } \\
\text { CAGTTGTCTAATTCCAG-3' }\end{array}$ \\
\hline ASC & $\begin{array}{l}5^{\prime}-\text { CACAAATCAGTCTC- } \\
\text { CAACACC- } 3^{\prime}\end{array}$ & $\begin{array}{l}\text { 5'-TAACCATTACCTTGT- } \\
\text { TCCCA-3' }\end{array}$ \\
\hline Caspase-1 & $\begin{array}{l}5^{\prime} \text {-CACTCGTA- } \\
\text { CACCTCTTGCCCTC-3' }\end{array}$ & $\begin{array}{l}5^{\prime} \text {-CTTTCACCTCTTTCAC- } \\
\text { CATCTCCA-3' }\end{array}$ \\
\hline TLR4 & $\begin{array}{l}\text { 5'-TGAGTGGTCAGTGT- } \\
\text { GATTGTGGT-3' }\end{array}$ & $\begin{array}{l}\text { 5'-TGTAGTGAAGGCAGAG- } \\
\text { GTGAAAG-3' }\end{array}$ \\
\hline $\mathrm{BAX}$ & $\begin{array}{l}5^{\prime}-\mathrm{GCAAACTGGTGCTCAA-} \\
\text { GG-3' }\end{array}$ & $\begin{array}{l}5^{\prime}-C A G C C A C A A A G A T G- \\
\text { GTCA-3' }\end{array}$ \\
\hline $\mathrm{BCL} 2$ & $\begin{array}{l}\text { 5'-ACTTTTAGGCGTGGCT- } \\
\text { GATG-3' }\end{array}$ & $\begin{array}{l}5^{\prime} \text {-GTGCTGCTCACTGTAT- } \\
\text { TTTATTTT-3' }\end{array}$ \\
\hline TNF-a & $\begin{array}{l}\text { 5'-TGTCTCAGCCTCTTCT- } \\
\text { CATTCCTG-3' }\end{array}$ & $\begin{array}{l}5^{\prime} \text {-AGGCCATTTGGGAACT- } \\
\text { TCTCATCC-3' }\end{array}$ \\
\hline GADPH & $\begin{array}{l}\text { 5'-TGACATCAAGAAGGTG- } \\
\text { GTGAAG-3' }\end{array}$ & $\begin{array}{l}5^{\prime}-C G A A G G T G G A A G A G T- \\
\text { GGGAG-3' }\end{array}$ \\
\hline
\end{tabular}


(1:1000 dilution, Abcam, Cambridge, MA, USA). The samples were incubated with secondary antibody (1:500 dilution, Abcam, Cambridge, MA, USA) for $2 \mathrm{~h}$ at $37^{\circ} \mathrm{C}$ and then the cells' nuclei were stained with PI (1:1000, Sigma-Aldrich, St. Louis, MO, USA). The cell counting and merging of the pictures were performed by Image J software (Image J U. S. National Institutes of Health, Bethesda, MA, USA).

\section{Statistical Analysis}

The collected data were analyzed using SPSS version 20.0 software. The values were tested for normality using the Kolmogorov-Smirnov test. The statistical significance of the results was determined using the independent samples $t$-test and Mann-Whitney $U$ test. The results were presented as the mean \pm standard deviation $(\mathrm{SD})$ and $P \leq 0.05$ was considered as statistically significant.

\section{Results}

\section{Histological Assessment}

Histological analysis of ovarian follicles was assessed with H\&E staining (Fig. 1). The results showed that percentage of primordial follicles in the maternal separation group (17.226 \pm 9.096) was significantly lower compared with the control group $(53.188 \pm 4.485)(P<0.001)$. There was no significant difference between percentage of primary follicles in the maternal separation group $(5.584 \pm 3.229)$ and percentage of primary follicles in the control group $(5.839 \pm 2.387)$ $(P>0.05)$. Percentage of secondary follicles in the maternal separation group $(28.247 \pm 5.621)$ was significantly higher compared with the control group $(16.899 \pm 5.639, P<0.01)$. In addition, percentage of Graafian follicles in the maternal separation group $(48.941 \pm 7.826)$ was significantly higher coms pared with the control group (24.072 $\pm 1.908, P<0.001$, Fig. 2$)$.

\section{ROS Evaluation}

Reactive oxygen species production in ovarian tissue was detected using the DCFH-DA assay. The level of ROS production in ovarian tissue of the maternal separation group $(614.4183 \pm 28.6545)$ was significantly higher compared with the control group (363.9886 $\pm 48.3404, P<0.01$, Fig. 3$)$.

\section{RT-qPCR Analysis}

The results of RT-qPCR analysis showed that expression of the following significantly increased in the maternal separation group compared with the control group: TNF- $\alpha(P<0.001)$, NLRP3, ASC, caspase-1, TLR4 and BAX $(P<0.01)$. In contrast, the expression of BCL2 gene significantly decreased in the maternal separation group compared with the control group $(P<0.01)$ (Fig. 4)

\section{Enzyme-linked Immunosorbent Assa}

Enzyme-linked immunosorbent assay results showed that the level concentrations of ATP and GPx were significantly lower in the maternal separation group compared with the control group $(2.4992 \pm 0.03$ vs. $3.03497 \pm 0.02, P<0.001$ and 147.46 \pm 11.58 vs. $399.3414 \pm 23.39, P<0.001$ respectively). The IL- $1 \beta$ and IL-18 concentrations in the maternal separation group were higher than the control group $(2.4773 \pm 0.01$ vs. $1.8753 \pm$

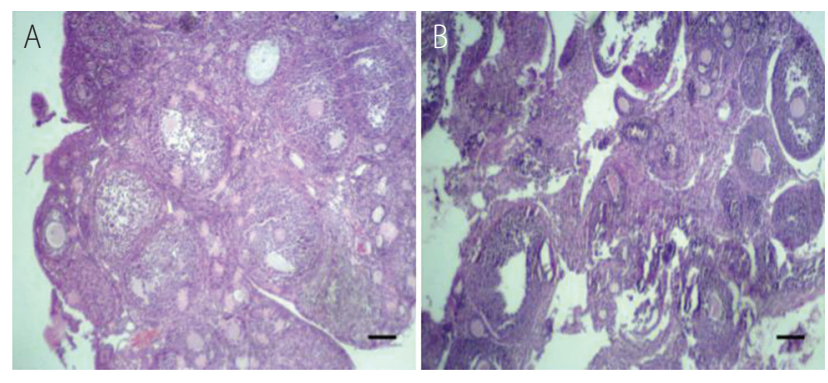

Fig. 1 The histopathological features provided from H\&E-stained ovarian sections in the control (A) and the maternal separation (B) groups. Scale bars are $20 \mu \mathrm{m}$. Samples were analyzed in triplicate.

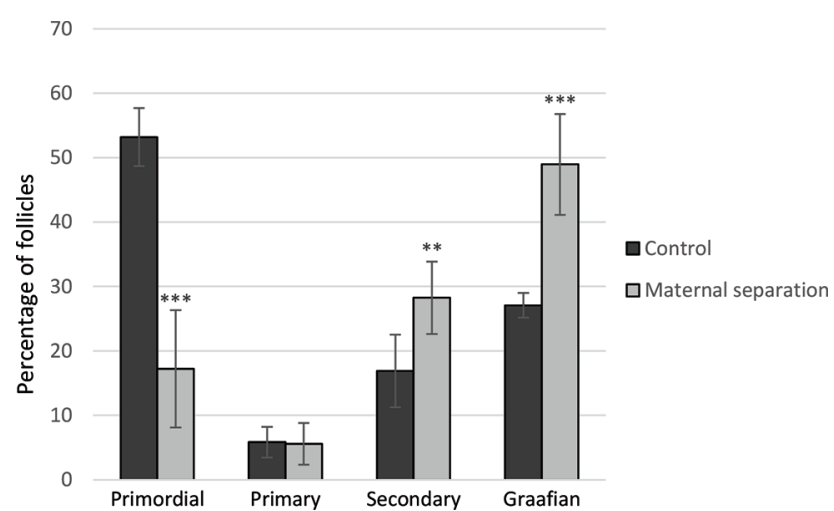

Fig. 2 Effect of maternal separation on mice ovarian follicles. Values are reported as mean \pm SD. ${ }^{* *} P<0.01,{ }^{* * *} P<0.001$. Samples were analyzed in triplicate.

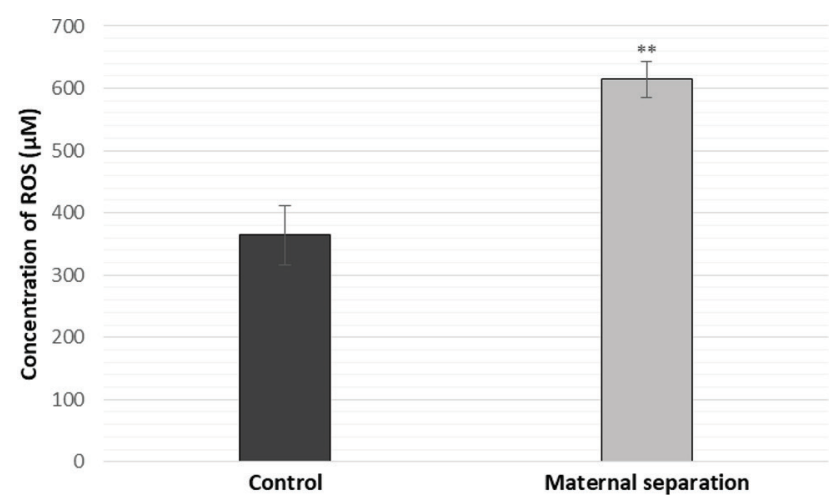

Fig. 3 The level concentrations of ROS production in the ovary. Values are reported as mean $\pm S D$. ${ }^{* *} p<0.01$. Samples were analyzed in triplicate. ROS, reactive oxygen species; SD, standard deviation.

0.02 and $3.3494 \pm 0.04$ vs. $1.9481 \pm 0.08$ respectively, $P<0.001$ for both) (Fig. 5).

\section{Immunocytochemical Analysis}

Caspase-3 and NLRP3 markers were used to label the ovarian cells in the control and maternal separation groups. The nuclei were stained with PI (Fig. 6). Immunocytochemical analysis showed that the mean percentage of caspase- 3 positive cells in the maternal separation group was significantly higher compared with the control group (50.6666 $\pm 1.76 \%$ vs. $24.00 \pm$ $0.58 \%, P<0.001)$. Also, the mean percentage of NLRP3 positive cells was significantly higher in the maternal separation group 


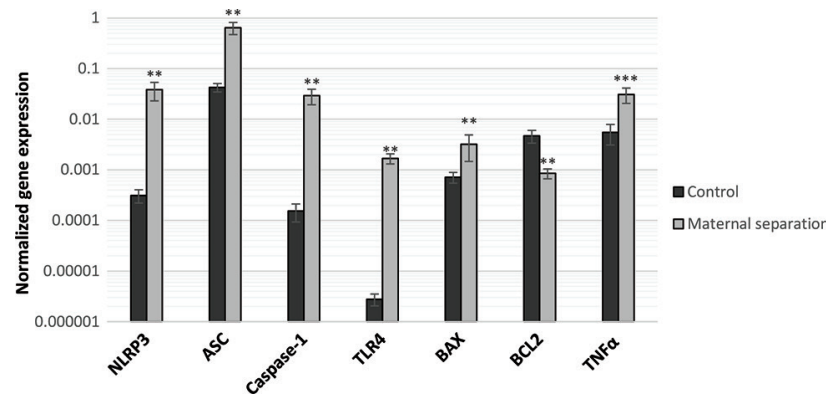

Fig. 4 The gene expression of NLRP3, ASC, caspase-1, TLR4, BAX, BCL2 and TNF- $a$ using RT-qPCR. Values are reported as mean \pm SD. ${ }^{* * P}<0.01,{ }^{* *} P<0.001$. Samples were analyzed in triplicate. SD, standard deviation; RT-qPCR, real-time reverse transcription polymerase chain reaction; ASC, apoptosis-associated speck-like protein containing a CARD.

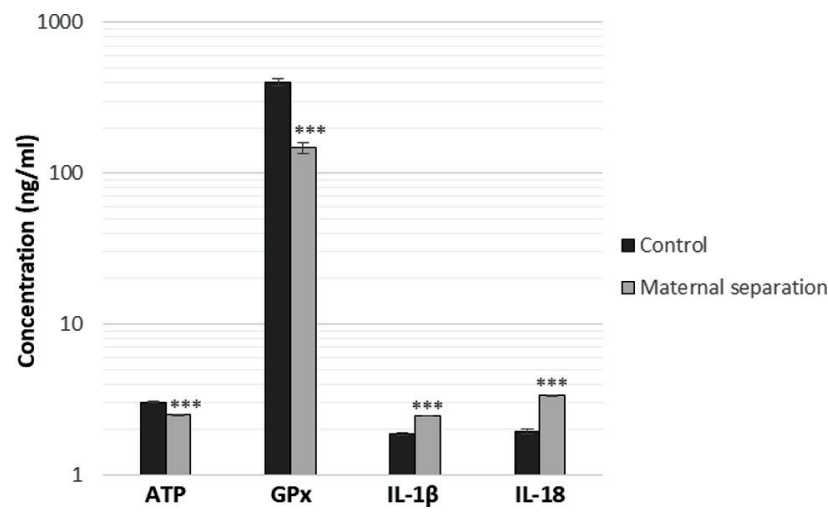

Fig. 5 The level concentrations of ATP, GPx, IL-1 $\beta$ and IL-18 measured using ELISA. Data were analyzed using the Mann-Whitney $U$ test. Values are reported as mean \pm SD. ${ }^{* * *} \boldsymbol{P}<\mathbf{0 . 0 0 1}$. Samples were analyzed in triplicate. ELISA, enzyme-linked immunosorbent assay.
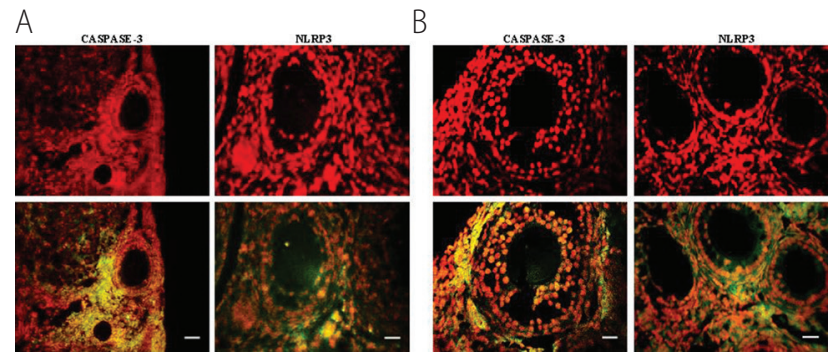

Fig. 6 Immunocytochemical analysis of ovarian cells for caspase-3 and NLRP3 markers. (A) Control group; (B) maternal separation group; upper panel: PI stained pictures; lower panel: merged pictures of $\mathrm{PI}$ and secondary antibody stained cells. Scale bars are $10 \mu \mathrm{m}$. Samples were analyzed in triplicate.

in compared with the control group (44.00 $\pm 1.53 \%$ vs. 20.6666 $\pm 1.45 \%, P<0.001$ ) (Fig. 7).

\section{Discussion}

The findings of this study showed that maternal separation stress led to significant histological alterations in the ovarian tissue, including decreased percentage of primordial follicles and increased percentage of secondary and Graafian follicles. In addition, maternal separation stress during early life increased ROS production and decreased ATP and GPx concentrations. Furthermore, expression of cytokines and genes involved in

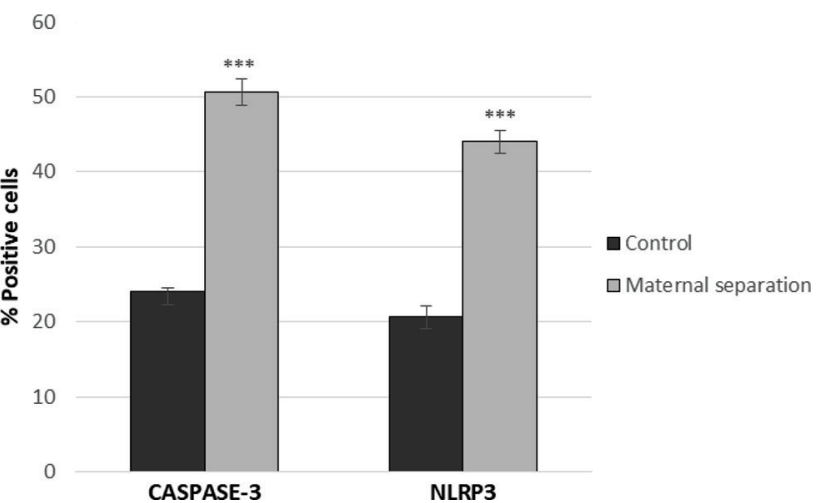

Fig. 7 Comparison of the mean percentage of positive cells for caspase- 3 and NLRP3 markers by immunocytochemical assessment. Values are reported as mean \pm SD. ${ }^{* * *} P<0.001$. Samples were analyzed in triplicate.

inflammation and apoptosis including NLRP3, ASC, caspase-1, TLR4, TNF- $\alpha$, IL-1 $\beta$, IL-18, BAX and BCL2 were significantly affected in the maternal separation group. Our results also showed that maternal separation stress significantly increased percentage of caspase-3 and NLRP3 positive cells.

According to a large body of evidence, maternal separation has long-lasting effects on neurodevelopmental and behavioral health that can increase susceptibility to psychopathology in adulthood. ${ }^{30}$ It is known that the function of HPA axis, an essential hormonal response system to manage stress, adversely affected by maternal separation stress. ${ }^{31}$ Considering the regulatory role of HPA axis in many homeostatic systems, these adverse modifications in HPA axis activity can result in functional alterations in the biological systems such as neuroendocrine, immune and reproductive systems. ${ }^{3,5,32,33}$ Furthermore, stress can affect the function of the adrenergic system that change the activities of the endocrine and immune systems. ${ }^{3}$ Cross-talk between the endocrine and immune systems is necessary for normal development and function of the female reproductive system. ${ }^{4}$ Although it remains unknown how innate immune function is stimulated by stress, the role of different factors including glucocorticoids, danger signals and catecholamines are evidenced by several studies. ${ }^{7-10}$

Previous studies have indicated that NLRs play a regulatory role in the reproductive and innate immune systems in mammals. ${ }^{34}$ The NLRP3 inflammasome, as a well-known NLR, is a cytoplasmic complex which is activated by danger signals derived from pathogens and metabolic dysregulation. Activation of NLRP3 inflammasome lead to regulating the secretion of the pro-inflammatory cytokines IL- $1 \beta$ and IL- $18 .{ }^{35}$

Our findings showed that stress induced by maternal separation significantly affect the NLRP3 inflammasome components and inflammatory molecules including NLRP3, ASC, caspase-1, TLR4, TNF- $\alpha$, IL1- $\beta$, IL18- and caspase- 3 . Furthermore, maternal separation stress can significantly affect mitochondrial activation and resulted in increase of ROS production and decrease of ATP level. In addition, this chronic stress can affect the mRNA expression of BCL2 and BAX genes which are involved in apoptosis pathways.

It is well known that increased ROS production is associated with mitochondrial dysfunction and apoptosis. Recently, it has been identified that mitochondria play a key role in activation of NLRP3 inflammasome. ${ }^{36}$ Mitochondrial damage can trigger signals of apoptosis and induce NLRP3 inflammasome 
activation through release of oxidized mitochondrial DNA into cytosol. ${ }^{36,37}$ Furthermore, the findings of studies indicated that mitochondrial ROS and DNA play an important role in NLRP3 inflammasome activation. ${ }^{38,39}$ In addition to its role in apoptosis, it has been suggested that BCL2 might play a role in the inhibition of NLRP3 inflammasome activation. Shimada et al. have described that increased expression of BCL2 lead to decrease in IL- $1 \beta$ levels. From this, it can be concluded that apoptosis plays a role in NLRP3 inflammasome activation. ${ }^{36}$ In addition, recently some studies have described that TNF, as a proinflammatory cytokine, can regulate the NLRP3 inflammasome activation. ${ }^{40,41}$ Given these data and our findings, plus the known effects of stress on the neuroendocrine system, it seems that maternal separation stress can affect the NLRP3 inflammasome components and inflammatory molecules with alteration in level of ROS, BCL 2 and TNF- $\alpha$ and subsequently lead to increase of IL1- $\beta$ and IL18- production.

Our findings showed that maternal separation resulted in histological alterations in the ovarian tissue, including decreased percentage of primordial follicles and increased percentage of secondary and Graafian follicles. In addition to reducing the number of primary follicles, stress likely accelerates the process of evolution of primary follicles to secondary and Graafian follicles. It seems that maternal separation stress lead to fast down the folliculogenesis process that may reduce the ovarian follicular reserve and induce a shorter reproductive lifespan.

In our study, maternal separation stress cause to increase of ROS production and decrease of GPx concentration. Cells have defensive strategies to prevent oxidative injury caused by excessive production of ROS, such as anti-oxidant enzymes like catalase (CAT), superoxide dismutase and GPx. ${ }^{42}$ Although ROS play physiological roles during folliculogenesis process, maturation of oocyte, and fertilization, ${ }^{43}$ increased ROS production and oxidative stress can suppress the antioxidant defense and influences the fertilization capacity. ${ }^{44}$ The findings of studies indicated that oxidative stress lead to follicular atresia and ovarian follicle aging. ${ }^{43,45,46}$ Furthermore, excessive production of ROS and oxidative stress can lead to pathologic events in the ovary including inflammation and apoptosis. ${ }^{47}$
Findings of studies described that IL-1 is expressed in the mammalian ovary and play a physiological role in ovary. Although inflammation is very important in reproductive processes such as ovulation, menstruation and implantation, uncontrolled inflammation has adverse effects on normal function of ovary. ${ }^{48,49}$ Uri-Belapolsky et al. ${ }^{50}$ have reported that IL-1 may enhance the inflammatory genes expression and promote apoptotic signaling pathways that leads to exhaust the ovarian reserve. On the other hand, it is well known that follicular atresia can occur via mitochondrial pathway or binding of death receptors to their ligands such as TNF- $\alpha$ and Fas. ${ }^{51}$ According to the above-mentioned data and the results of this study, we can conclude that maternal separation stress via activation of inflammatory and apoptosis pathways may have adverse effects on folliculogenesis process and the number of ovarian follicles.

\section{Conclusion}

This study provides a glimpse of the effects of maternal separation stress on ovarian tissue in female mice as adults. Based on this study, maternal separation stress had detrimental effects on ovarian tissue, in addition to the known effects of stress on the HPA axis and neuroendocrine system, probably through increase of ROS production and impact on mitochondrial function, inflammatory process and apoptosis pathways. An improved insight into the mechanisms by which maternal separation stress affects the reproductive system may provide necessary information to keep the reproductive system healthy.

\section{Acknowledgments}

This research has been funded by Tehran University of Medical Sciences (TUMS); grant no. 32229. The authors thank Tehran University of Medical Sciences for its support.

\section{Conflict of Interest}

The authors declare that there is no known conflict of interest regarding this publication.

\section{References}

1. Tractenberg SG, Levandowski ML, de Azeredo LA, Orso R, Roithmann LG, Hoffmann ES, et al. An overview of maternal separation effects on behavioural outcomes in mice: evidence from a four-stage methodological systematic review. Neurosci Biobehav Rev. 2016;68:489-503.

2. Harrison EL, Baune BT. Modulation of early stress-induced neurobiological changes: a review of behavioural and pharmacological interventions in animal models. Transl Psychiatry. 2014;4:e390.

3. Jóźków P, Mędraś M. Psychological stress and the function of male gonads. Endokrynol Pol. 2012;63:44-49

4. Ostanin AA, Aizikovich Bl, Aizikovich IV, Kozhin AY, Chernykh ER. Role of cytokines in the regulation of reproductive function. Bull Exp Biol Med. 2007;143:75-79

5. Fleshner M. Stress-evoked sterile inflammation, danger associated molecular patterns (DAMPs), microbial associated molecular patterns (MAMPs) and the inflammasome. Brain Behav Immun. 2013;27:1-7.

6. Rock KL, Latz E, Ontiveros F, Kono H. The sterile inflammatory response. Annu Rev Immunol. 2009:28:321-342.

7. Johnson JD, Campisi J, Sharkey CM, Kennedy SL, Nickerson M, Greenwood $\mathrm{BN}$, et al. Catecholamines mediate stress-induced increases in peripheral and central inflammatory cytokines. Neuroscience. 2005;35:1295-1307.

8. Mazzeo RS, Donovan D, Fleshner M, Butterfield GE, Zamudio S, Wolfel EE, et al. Interleukin-6 response to exercise and high-altitude exposure: influence of a-adrenergic blockade. J Appl Physiol. 2001;91:2143-2149.

9. Frank MG, Thompson BM, Watkins LR, Maier SF. Glucocorticoids mediate stress-induced priming of microglial pro-inflammatory responses. Brain Behav Immun. 2012;26:337-345.

10. Campisi J, Sharkey C, Johnson JD, Asea A, MaslanikT, Bernstein-Hanley I, et al. Stress-induced facilitation of host response to bacterial challenge in F344 rats is dependent on extracellular heat shock protein 72 and independent of alpha beta T cells. Stress. 2012;15:637-646.

11. Gómez López M, Domínguez López A, Abarca Rojano E, Rojas Hernández S, Martínez Godínez Mde L, Miliar García A, et al. 17ß-Estradiol transcriptionally modulates NIrp1 and NIrp3 inflammasomes in gonadectomized rats with inflammation. Immunopharmacol Immunotoxicol. 2015;37:343-350.

12. Bazrafkan M, Nikmehr B, Shahverdi A, Hosseini, Hassani F, Poorhassan M, et al. Lipid peroxidation and its role in the expression of NLRP1a and NLRP3 genes in testicular tissue of male rats: a model of spinal cord injury. Iran Biomed J. 2018:22:151-159.

13. Nikmehr B, Bazrafkan M, Hassanzadeh G, Shahverdi A, Sadighi Gilani MA, Kiani S, et al. The correlation of gene expression of inflammasome indicators and impaired fertility in rat model of spinal cord injury: a time course study. Urol J. 2017;14:5057-5063.

14. Mohamadi Y, Noori Moghahi SMH, Mousavi M, Borhani-Haghighi M, Abolhassani F, Kashani IR, et al. Intrathecal transplantation of Wharton's jelly mesenchymal stem cells suppresses the NLRP1 inflammasome in the rat model of spinal cord injury. J Chem Neuroanat. 2019;97:1-8. 
15. Yang CA, Chiang BL. Inflammasomes and human autoimmunity: a comprehensive review. J Autoimmun. 2015:61:1-8.

16. Jin C, Flavell RA. Molecular mechanism of NLRP3 inflammasome activation. J Clin Immunol. 2010;30:628-631.

17. Ghaffari, N., Hassanzadeh, G., Nowrouzi, A., Gholaminejhad, M., Mokhtari, T., Seifali, R.... \& Akbari, M. (2018). Antioxidative and anti-inflammatory effects of Cichorium intybus L. seed extract in ischemia/reperfusion injury model of rat spinal cord. Journal of Contemporary Medical Sciences, 4(4).

18. Shalini S, Dorstyn L, Dawar S, Kumar S. Old, new and emerging functions of caspases. Cell Death Differ. 2015;22:526-539.

19. Wagenmaker ER, Breen KM, Oakley AE, Tilbrook AJ, Karsch FJ. Psychosocial stress inhibits amplitude of gonadotropin-releasing hormone pulses independent of cortisol action on the type II glucocorticoid receptor. Endocrinology. 2009;150:762-769.

20. Keating DJ. Mitochondrial dysfunction, oxidative stress, regulation of exocytosis and their relevance to neurodegenerative diseases. J Neurochem. 2008;104:298-305.

21. Mailloux RJ. Mitochondrial antioxidants and the maintenance of cellular hydrogen peroxide levels. Oxid Med Cell Longev. 2018;2018:7857251.

22. Madrigal JL, Olivenza R, Moro MA, Lizasoain I, Lorenzo P, Rodrigo J, et al. Glutathione depletion, lipid peroxidation and mitochondrial dysfunction are induced by chronic stress in rat brain. Neuropsychopharmacology 2001;24:420-429.

23. Cecchino GN, Seli E, Alves da Motta EL, García-Velasco JA. The role of mitochondrial activity in female fertility and assisted reproductive technologies: overview and current insights. Reprod Biomed Online. 2018:36:686-697.

24. Agarwal A, Aponte-Mellado A, Premkumar BJ, Shaman A, Gupta S. The effects of oxidative stress on female reproduction: a review. Reprod Biol Endocrinol. 2012;10:49.

25. Amini-Khoei H, Amiri S, Shirzadian A, Haj-Mirzaian A, Alijanpour S, Rahimi-Balaei $\mathrm{M}$, et al. Experiencing neonatal maternal separation increased the seizure threshold in adult male mice: involvement of the opioid system. Epilepsy Behav. 2015;52:37-41

26. Amini-Khoei H, Amiri S, Mohammadi-Asl A, Alijanpour S, Poursaman S, Haj-Mirzaian A, et al. Experiencing neonatal maternal separation increased pain sensitivity in adult male mice: involvement of oxytocinergic system. Neuropeptides. 2017:61:77-85.

27. Amini-Khoei H, Haghani-Samani E, Beigi M, Soltani A, Mobini GR, Balali-Dehkordi S, et al. On the role of corticosterone in behavioral disorders, microbiota composition alteration and neuroimmune response in adult male mice subjected to maternal separation stress. Int Immunopharmacol. 2019;66:242-50

28. Dym M, Jia MC, Dirami G, Price JM, Rabin SJ, Mocchetti I, Ravindranath N. Expression of c-kit receptor and its autophosphorylation in immature rat type A spermatogonia. Biol Reprod. 1995;52:8-19.

29. Fatemi N, Sanati MH, Shamsara M, Moayer F, Zavarehei MJ, Pouya A, et al. TBHP-induced oxidative stress alters microRNAs expression in mouse testis. J Assist Reprod Genet. 2014;31:1287-1293.

30. Slotten HA, Kalinichev M, Hagan JJ, Marsden CA, Fone KC. Long-lasting changes in behavioural and neuroendocrine indices in the rat following neonatal maternal separation: gender-dependent effects. Brain Res. 2006;1097:123-132

31. Clarke AS. Social rearing effects on HPA axis activity over early development and in response to stress in rhesus monkeys. Dev Psychobiol. 1993;26:433-446.

32. Kuhn CM, Schanberg SM. Responses to maternal separation: mechanisms and mediators. Int J Dev Neurosci. 1998;16:261-270.
33. G M. Central nervous regulation of the hypothalamic-pituitary-adrenal axis and its impact on fertility, immunity, metabolism and animal welfare-a review. Arch Anim Breed. 2002;45:575-595.

34. Peng $\mathrm{H}$, Zhang W, Xiao T, Zhang Y. Nlrp4g is an oocyte-specific gene but is not required for oocyte maturation in the mouse. Reprod Fertil Dev. 2014;26:758-768

35. Tschopp J, Schroder K. NLRP3 inflammasome activation: the convergence of multiple signalling pathways on ROS production? Nat Rev Immunol. 2010;10:210-215.

36. Shimada K, Crother TR, Karlin J, Dagvadorj J, Chiba N, Chen S, et al. Oxidized mitochondrial DNA activates the NLRP3 inflammasome during apoptosis. Immunity. 2012;36:401-414

37. Shimada K, Crother TR, Karlin J, Chen S, Chiba N, Ramanujan VK, Vergnes L, Ojcius DM, Arditi M. Caspase-1 dependent IL-1 $\beta$ secretion is critical for host defense in a mouse model of Chlamydia pneumoniae lung infection. PLoS One. 2011;6:e21477.

38. Zhou R, Yazdi AS, Menu P, Tschopp J. A role for mitochondria in NLRP3 inflammasome activation. Nature 2011:469:221-225.

39. Nakahira K, Haspel JA, Rathinam VA, Lee SJ, Dolinay T, Lam HC, et al. Autophagy proteins regulate innate immune responses by inhibiting the release of mitochondrial DNA mediated by the NALP3 inflammasome. Nat Immunol. 2011;12:222-230.

40. Wree A, McGeough MD, Inzaugarat ME, Eguchi A, Schuster S, Johnson CD, et al. NLRP3 inflammasome driven liver injury and fibrosis: roles of IL-17 and TNF in mice. Hepatology. 2018;67:736-749.

41. McGeough MD, Wree A, Inzaugarat ME, Haimovich A, Johnson CD, Peña CA, et al. TNF regulates transcription of NLRP3 inflammasome components and inflammatory molecules in cryopyrinopathies. J Clin Invest. 2017;127: 4488-4497.

42. Ighodaro OM, Akinloye OA. First line defence antioxidants-superoxide dismutase (SOD), catalase (CAT) and glutathione peroxidase (GPX): their fundamental role in the entire antioxidant defence grid. Alexandria J Med. 2018:54:287-293.

43. Agarwal A, Gupta S, Sekhon L, Shah R. Redox considerations in female reproductive function and assisted reproduction: from molecular mechanisms to health implications. Antioxid Redox Signal. 2008;10: 1375-1403.

44. Agarwal A, Virk G, Ong C, du Plessis SS. Effect of oxidative stress on male reproduction. World J Mens Health. 2014;32:1-17

45. Lim J, Luderer U. Oxidative damage increases and antioxidant gene expression decreases with aging in the mouse ovary. Biol Reprod. 2011;84:775-782.

46. Tatone C, Amicarelli F, Carbone MC, Monteleone P, Caserta D, Marci R, et al. Cellular and molecular aspects of ovarian follicle ageing. Hum Reprod Update. 2008;14:131-142.

47. Behrman HR, Kodaman PH, Preston SL, Gao S. Oxidative stress and the ovary. J Soc Gynecol Investig. 2001:8:S40-S42.

48. Jabbour HN, Sales KJ, Catalano RD, Norman JE. Inflammatory pathways in female reproductive health and disease. Reproduction. 2009;138:903-919.

49. Herath S, Williams EJ, Lilly ST, Gilbert RO, Dobson H, Bryant CE, et al. Ovarian follicular cells have innate immune capabilities that modulate their endocrine function. Reproduction. 2007;134:683-693.

50. Uri-Belapolsky S, Shaish A, Eliyahu E, Grossman H, Levi M, Chuderland D, et al. Interleukin-1 deficiency prolongs ovarian lifespan in mice. Proc Nat Acad Sci U S A. 2014;111:12492-12497.

51. Hussein MR. Apoptosis in the ovary: molecular mechanisms. Hum Reprod Update. 2005;11:162-77 